\title{
Synthetic emmprin peptides inhibit tumor cell- fibroblast interaction-stimulated upregulation of MMP-2 and tumor cell invasion
}

\author{
KAORI KOGA ${ }^{1}$, MIKIKO AOKI ${ }^{2}$, TETSURO SAMESHIMA ${ }^{3}$, MAKOTO HAMASAKI $^{1}$, NAGAYASU EGAWA ${ }^{4}$, \\ MOTOHARU SEIKI ${ }^{5}$, BRYAN P. TOOLE ${ }^{7}$, JUNJI SUZUMIYA ${ }^{6}$ and KAZUKI NABESHIMA ${ }^{1,2}$ \\ Departments of Pathology, ${ }^{1}$ Fukuoka University Hospital and ${ }^{2}$ School of Medicine, 7-45-1 Nanakuma, Jonan-ku, \\ Fukuoka 814-0180; ${ }^{3}$ Department of Neurosurgery, NTT Medical Center Tokyo, 5-9-22 Higashi-Gotanda, Shinagawa-ku, \\ Tokyo 141-8625; ${ }^{4}$ Virology Division, National Cancer Center Research Institute, 5-1-1 Tsukiji, Chuo-ku, \\ Tokyo 104-0045; ${ }^{5}$ Division of Cancer Cell Research, Institute of Medical Science, the University of Tokyo, \\ 4-6-1 Shirokanedai, Minato-ku, Tokyo 108-8639; ${ }^{6}$ Shimane University Hospital Cancer Center, 89-1 Enya, \\ Izumo, Shimane 693-8501, Japan; ${ }^{7}$ Department of Regenerative Medicine and Cell Biology, \\ Medical University of South Carolina, 171 Ashley Avenue, Charleston, SC 29403, USA
}

Received March 15, 2011; Accepted May 17, 2011

DOI: $10.3892 /$ ijo.2011.1060

\begin{abstract}
Stromal cells are the main source of matrix metalloproteinases (MMPs) in human carcinoma tissues. Emmprin is a glycosylated transmembrane protein containing two immunoglobulin (Ig) domains that is expressed in carcinoma cells and stimulates MMP production by adjacent stromal cells. The first Ig domain (ECI) of emmprin contains the biologically active site. We investigated whether synthetic peptides carrying a partial ECI sequence could inhibit emmprin activity. Only the second peptide (emp\#2), which contains a putative N-glycosylation site sequence, inhibited emmprin-stimulated production of MMP-2 in co-cultures of fibroblasts and several different human tumor cells types, including carcinoma, sarcoma, melanoma, leukemia and glioma cells. Moreover, emp\#2 significantly inhibited the invasive activity of glioblastoma cells promoted by interaction with fibroblasts. Perturbation of emmprin activity by this peptide may have potential therapeutic uses in the prevention of MMP-2 dependent cancer invasion.
\end{abstract}

\section{Introduction}

Tumor cell invasion and metastasis requires degradation of extracellular matrix, which is mainly achieved by various proteolytic enzymes including zinc-dependent matrix metalloproteinases (MMPs) (1). In situ hybridization studies of human surgical specimens have shown that stromal fibroblasts

Correspondence to: Dr Kazuki Nabeshima, Department of Pathology, Fukuoka University School of Medicine, 7-45-1 Nanakuma, Jonan-ku, Fukuoka 814-0180, Japan

E-mail: kaznabes@fukuoka-u.ac.jp

Key words: emmprin, MMPs, tumor invasion, fibroblast interaction, inhibitory peptide, cell-cell interaction, glycosylation, peptide are the predominant source of MMP-2 in most carcinomas (2-4). Carcinoma cells stimulate nearby fibroblasts to produce MMPs via soluble cytokines and growth factors, or by cell surface interactions mediated by plasma membrane proteins, such as emmprin (5-7). Emmprin, also called basigin/CD147, is expressed in significantly higher levels in tumor cells than in normal cells. Emmprin stimulates MMP production in fibroblasts, and hence the elevated expression of emmprin on the surface of tumor cells could promote tumor progression by inducing MMP production in peritumoral fibroblasts $(8,9)$. It was also shown recently that emmprin also mediates angiogenesis via stimulation of vascular endothelial growth factor (VEGF) (10), tumor cell glycolysis $(11,12)$, and multidrug resistance (13-16). A role for emmprin in epithelial-mesenchymal interactions was also reported (17).

Emmprin is an integral plasma membrane glycoprotein of the immunoglobulin (Ig) superfamily that contains two extracellular Ig domains (18). The activity-blocking monoclonal antibody, E11F4, recognizes the first Ig domain (ECI) of emmprin, implicating this region is involved in the various emmprin activities (18). Furthermore, recombinant and unglycosylated emmprin produced by bacteria is smaller in molecular size and in inactive, whereas emmprin isolated from Chinese hamster ovary cells is highly glycosylated with a molecular mass of approximately $58 \mathrm{kDa}$, similar to the native protein. The hamster emmprin also successfully stimulated production of MMP-1, -2 , and -3 from fibroblasts (19). The presence of a putative receptor for emmprin on the fibroblast cell membrane is postulated, although not yet proven (20).

Based on the above findings, the present study tested the hypothesis that synthetic peptides carrying the ECI sequences can modify or regulate emmprin activity. The results showed that one of the ECI-derived peptides tested, emp\#2, effectively inhibited emmprin-induced stimulation of MMP-2 production. This peptide contains a putative $\mathrm{N}$-glycosylation 
site, but shows no glycosylation. Furthermore, emp\#2 peptide effectively inhibited the enhanced invasion of matrigel by glioblastoma cells in the co-presence of fibroblasts.

\section{Materials and methods}

Cell culture. The human colon cancer cell line RCM-1 and the gastric cancer cell line TMK-1 were gifts from Professor H. Kataoka (Miyazaki University, Miyazaki, Japan) and Professor E. Tahara (Hiroshima University, Hiroshima, Japan), respectively. The human melanoma cell line A375, and the HTLV-1-transformed human T-cell line MT-2 (21) were purchased from the American Type Culture Collection (Manassas, VA), and the glioblastoma cell line U251 was obtained from the RIKEN cell bank (Tsukuba, Japan). FU-EPS-1 (22) was established from a patient with epithelioid sarcoma who had not received any chemotherapy before surgical resection. The human dermal fibroblast ST353 was obtained from non-lesional dermis around nodular fascitis. Human adult brain-derived fibroblast line, MBT-3, was obtained from a lung adenocarcinoma metastasis to the cerebellum. All cell lines were cultured in Dulbecco's modified Eagle's medium (DMEM) supplemented with $10 \%$ fetal bovine serum (FBS) and antibiotics (100 unit $/ \mathrm{ml}$ penicillin $\mathrm{G}$ and $100 \mu \mathrm{g} / \mathrm{ml}$ streptomycin) in a humidified atmosphere of $5 \% \mathrm{CO}_{2}$ at $37^{\circ} \mathrm{C}$.

Preparation and extraction of membranes. Emmprin was extracted from cell membranes as described previously (23). Tumor cells and fibroblasts were harvested from confluent cultures in $75-\mathrm{cm}^{2}$ culture bottles by mechanical scraping, and were then suspended and sonicated in $50 \mathrm{mM}$ Tris- $\mathrm{HCl}(\mathrm{pH}$ 7.4)/ $0.24 \mathrm{M}$ sucrose. The sonicated cell suspension was centrifuged at $500 \mathrm{~g}$ for $20 \mathrm{~min}$ and the supernatant was centrifuged at $100,000 \mathrm{~g}$ for $1 \mathrm{~h}$. The membrane pellet was extracted with $0.15 \mathrm{M} \mathrm{NaCl} / 0.5 \%$ Nonidet P-40/1 mM ethylenediaminetetraacetic acid (EDTA) $/ 2 \mathrm{mM}$ phenylmethylsulfonyl fluoride $/ 10 \mathrm{mM}$ Tris- $\mathrm{HCl}(\mathrm{pH} 8.2)$ at $4^{\circ} \mathrm{C}$ overnight. The extract was centrifuged at $100,000 \mathrm{~g}$ for $1 \mathrm{~h}$ and the supernatant was kept at $4^{\circ} \mathrm{C}$ until use. The presence of emmprin in the membrane extract was assayed using immunoblotting.

Stable knockdown of emmprin by RNA interference. To stably knock down the expression of emmprin, we used the BLOCK-iT Lentiviral RNAi Expression System (Invitrogen Corporation, Carlsbad, CA), as described previously (24). Gene-specific inserts were cloned into pLenti6 according to the manufacturer's instructions. The insert sequence for stable emmprin siRNA expression is 5'-CACAGTCTTCACTAC CGTAGCGAACTACGGTAGTGAAGACTGTGC. The lentivirus was produced in HEK 293FT cells using the ViraPower lentiviral expression system (Invitrogen), and the viruscontaining media were harvested for infection. Lentivirus expressing LacZ shRNA was used as a control. Stable shRNAexpressing cells were propagated and maintained in the presence of blasticidin (5 $\mu \mathrm{g} / \mathrm{ml}$; Invitrogen).

Peptides. Four peptides (emp\#1: AAGTVFTTVEDLGSKIL LTC, emp\#2: SLNDSATEVTGHRWLKGGVV, emp\#3: LKE DALPGQKTEFKVDSDDQWG, emp\#4: EYSCVFLPEPMG TANIQLHGPPR) were synthesized, each consisting of
20-23 amino acids (aa) and corresponding to about one fourth of the total ECI of the emmprin molecule (Fig. 1). Two peptides corresponding to the first and second halves of the emp\#2 peptide (emp\#2-1: SLNDSATEVT, emp\#2-2: GHRWLKGGVV) were also synthesized.

Emp\#1 was dissolved in dimethyl sulfoxide (DMSO). Other reagents were dissolved in PBS. The final concentration of DMSO in the culture medium was less than $0.01 \%$, and at this concentration the solvents alone had no deleterious effects on the cells or any detectable effects on MMP production.

Co-culture experiments. Co-culture experiments were done as previously described (5). Briefly, cultures containing fibroblasts, tumor cells (FU-EPS-1, A375, RCM-1, MT-2, U251) or both were established in $20 \mathrm{~mm}$ diameter wells containing $1.0 \mathrm{ml}$ DMEM with $10 \%$ FBS. Fibroblasts were incubated with tumor cells at a 1:1 ratio. The cells were allowed to attach for $24 \mathrm{~h}$ at $37^{\circ} \mathrm{C}$, after which the media were replaced with fresh serum-free (SF) DMEM containing $0.2 \%$ lactalbumin hydrolysate $(0.5 \mathrm{ml} /$ well) prior to the start of the experiment. Each experimental condition was carried out in duplicate or triplicate wells. Culture fluids were replaced with fresh SF DMEM at 3 days and harvested at 6 days. The harvested media were used for zymography and immunoblotting.

Inhibition experiments in co-cultures using antiemmprin blocking antibody (UM-8D6, Ancell Corporation, Bayport, MN) were performed as described previously (5). Furthermore, another inhibition experiments using TMK-1 cells, in which emmprin had been knocked down by shRNA transfections, was also performed.

For the co-culture experiments employing emp peptides (emp\#1-4), tumor cells were preincubated at $37^{\circ} \mathrm{C}$ for $45 \mathrm{~min}$ with 100,250 and $500 \mu \mathrm{g} / \mathrm{ml}$ of peptides before co-culturing with fibroblasts. Co-cultures were done in the presence of the peptides for 6 days with replacement of medium at 3 days, as described above.

Immunoblotting. Sodium dodecyl sulfate polyacrylamide gel electrophoresis (SDS-PAGE) of membrane extracts and conditioned medium was performed under reducing conditions using a $5-15 \%$ gradient gel (Biomate, Tokyo, Japan). After electrophoresis, the proteins were transferred electrophoretically to Immobilon membrane (Millipore, Bedford, MA). Non-specific sites were blocked with $5 \%$ non-fat dry milk in $0.05 \%$ Tween-20/Tris-buffered saline, $\mathrm{pH} 7.6$ (TBS-T) at $37^{\circ} \mathrm{C}$ for $1 \mathrm{~h}$ and the membrane was incubated overnight at $4^{\circ} \mathrm{C}$ with antibodies against emmprin [mouse monoclonal E11F4 (24) or 109403 (R\&D Systems, Flanders, NJ)], or MMP-2 (monoclonal 75-7F7, Daiichi Fine Chemical, Takaoka, Japan) dissolved in TBS-T containing $1 \%$ BSA. After washing with TBS-T, the membrane was incubated for $1 \mathrm{~h}$ with peroxidaseconjugated goat anti-mouse. The color was developed with chemiluminescence reagents (DuPont NEN, Boston, MA) according to the instructions supplied by the manufacturer. The bands on the film were subjected to image analysis (Adobe Photoshop, Adobe System, Mountain View, CA). Statistical analysis was performed using Student's t-test.

Zymography. Gelatinolytic activities in conditioned media were demonstrated using gelatin as a substrate, as described 
previously (5). SDS-PAGE was performed under non-reducing conditions using a $9 \%$ separating gel containing $1 \mathrm{mg} / \mathrm{ml}$ gelatin. After electrophoresis, the gel was shaken gently in detergent buffer $\left(5 \mathrm{mM} \mathrm{CaCl}_{2}, 2.5 \%\right.$ Triton X-100, and $50 \mathrm{mM}$ Tris- $\mathrm{HCl}, \mathrm{pH} 7.6$ ) at room temperature for $60 \mathrm{~min}$ to remove the SDS, and then incubated in reaction buffer $(0.15 \mathrm{M} \mathrm{NaCl}$, $10 \mathrm{mM} \mathrm{CaCl}_{2}, 0.02 \% \mathrm{NaN}_{3}$, and $50 \mathrm{mM}$ Tris- $\mathrm{HCl}, \mathrm{pH}$ 7.6) at $37^{\circ} \mathrm{C}$ for $30 \mathrm{~h}$ followed by staining with $2.5 \%$ Coomassie brilliant blue in $30 \%$ methanol and $10 \%$ acetate. Enzyme activity was detected as a clear band on the resulting blue background of undigested gelatin. Gel images were analyzed digitally (Adobe Photoshop) to quantitate relative activities.

In vitro invasion assay. The invasion assay was performed as described previously using a modified fluoroblock invasion assay (BD BioCoat Tumor Invasion system, BD Biosciences, Franklin Lakes, NJ) (25). Briefly, U251 glioblastoma cells grown to preconfluence in $25-\mathrm{cm}^{2}$ culture bottles were labeled with $10 \mathrm{ng} / \mathrm{ml}$ Dil (Invitrogen, Carlsbad, CA) in growth medium overnight. Labeled U251 cells were preincubated at $37^{\circ} \mathrm{C}$ for $45 \mathrm{~min}$ with 100 and $500 \mu \mathrm{g} / \mathrm{ml}$ of emp\#2 or control peptides before co-culturing with fibroblasts. The labeled U251 cells $\left(1.2 \times 10^{5} / \mathrm{ml}\right)$ and ST353 fibroblasts $\left(0.3 \times 10^{5} / \mathrm{ml}\right)$ were then placed in the upper compartment of a fluoroblock 24-multiwell insert plate, which was separated from the lower compartment by Matrigel $(25 \mu \mathrm{g})$-coated fluoroblock membrane of $8.0-\mu \mathrm{m}$ pore size. In both compartments, the incubation medium was SF DMEM/F12, supplemented with $0.1 \%$ bovine serum albumin (BSA). After incubation for $72 \mathrm{~h}$ at $37^{\circ} \mathrm{C}$ in a $5 \% \mathrm{CO}_{2}$ atmosphere, the labeled U251 cells that had invaded through the Matrigel were scanned by a bottom reading-type fluorescence plate reader (Labsystems Fluoroskan Ascent, Thermo Electron Co, Waltham, MA) at $590 \mathrm{~nm}$ (absorption/emission). Only the fluorescently labeled cells that migrated through the membrane were detected since the insert membrane blocks fluorescent light. Autofluorescence derived from wells containing medium only was subtracted from the inserts carrying the fluorescent cells. Means and SEMs were calculated and statistical differences were analyzed using Student's t-test for non-paired samples. Each assay was performed in triplicate and repeated two times with similar results.

\section{Results}

Involvement of emmprin in tumor cell-fibroblast interactioninduced upregulation of $M M P-2$. All six human tumor cell lines from various origins, including colon (RCM-1) and gastric (TMK-1) carcinoma, melanoma (A375), HTLV-1- transformed T-cells (MT-2), glioblastoma (U251), and epithelioid sarcoma (FUEPS-1), expressed emmprin (Fig. 2). On immunoblots of membrane extracts prepared from these cell lines, emmprin appeared as a broad band ranging from approximately 43 to $58 \mathrm{kDa}$.

To determine the involvement of emmprin in tumor cellfibroblast interaction-stimulated upregulation of MMP-2, we firstly performed co-culture experiments in the presence of emmprin activity-blocking antibody (UM-8D6). Co-cultures of U251 glioblastoma cells and fibroblasts stimulated the production of pro-MMP-2 by approximately 8.0 times compared with individual cultures, and this stimulation was

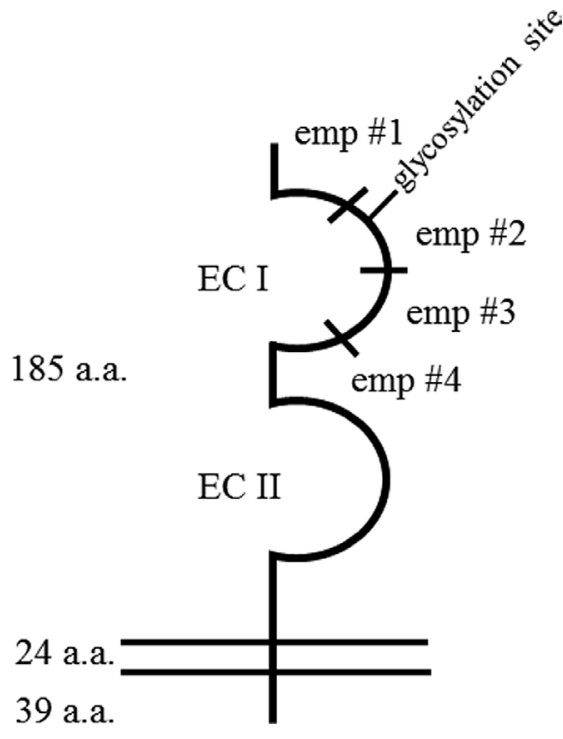

Figure 1. Molecular structure of the emmprin molecule and synthetic peptides. ECI, the first extracellular Ig domain; ECII, the second extracellular Ig domain; emp\#1-4, synthetic peptides carrying the partial sequences of ECI.

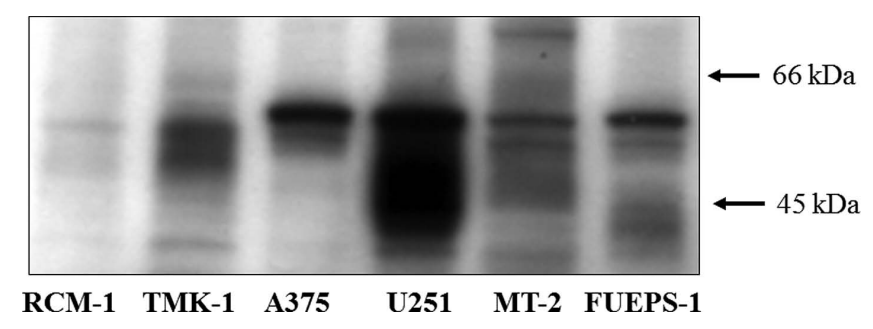

Figure 2. Expression of emmprin in six human tumor cell lines. Membrane extracts of each cell line prepared as described in Materials and methods were subjected to SDS-PAGE and immunoblotting with an anti-emmprin monoclonal antibody (E11F4). RCM-1, TMK-1, A375, U251, MT-2 and FU-EPS-1 are human tumor cell lines obtained from rectal and gastric carcinomas, melanoma, glioblastoma, HTLV-1 transformed T-cells, and epithelioid sarcoma, respectively.

inhibited by UM-8D6, i.e.. approximately $41.2 \%$ inhibition at $40 \mu \mathrm{g} / \mathrm{ml}$, while control non-immune IgG caused no inhibition (Fig. 3a). Similarly, stimulation of MMP-2 production in co-cultures of FUEPS-1 or MT-2 with fibroblasts was inhibited by the anti-emmprin antibody in a dose-dependent manner $(6,7)$.

Next, we used a retrovirus-based system to deliver shorthairpin RNA (shRNA) to knock down emmprin expression in TMK-1 cells. Introduction of shBSG lowered the emmprin expression level (Fig. 3b), which was unaffected by empty retrovirus (shLacz). Downregulation of emmprin by shBSG inhibited stimulation of MMP-2 production in co-cultures by approximately $45 \%$ compared with control TMK-1 cells (shLacz, Fig. 3c).

Effects of ECI-derived peptides on emmprin-mediated stimulation of MMP-2 production in co-cultures. Since a previous study (18) indicated that ECI contains the structure responsible for emmprin activity, we investigated whether ECI-derived peptides might act as competitive decoys. In this study, co-culture experi- 
(a)

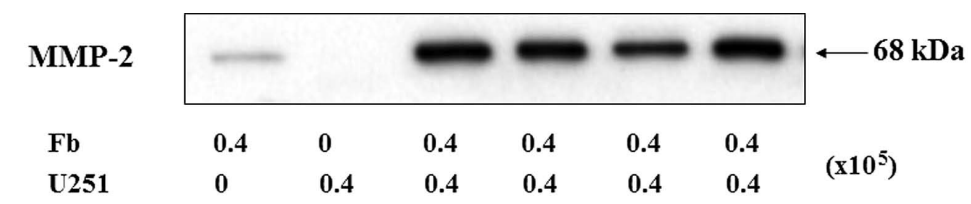

anti-emp Ab - $\quad-\quad-\quad 10 \quad 40 \quad-$

non-immune IgG -

(b) Emmprin E11/F4

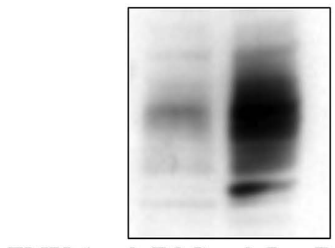

(c) MMP-2

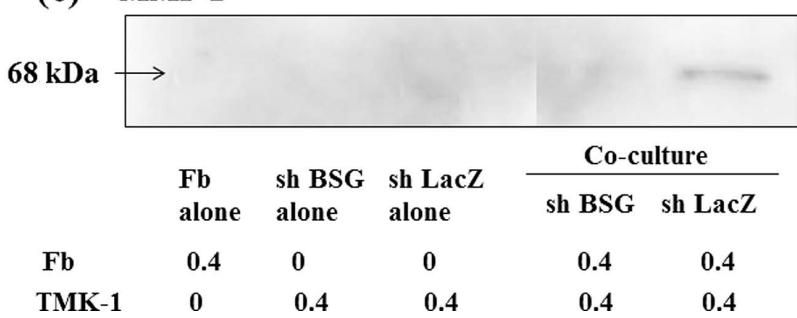

Figure 3. Involvement of emmprin in tumor cell-fibroblast interaction-stimulated upregulation of MMP-2 in co-cultures. (a) Co-culture experiments using U251 glioblastoma cells with ST353 fibroblasts were performed with emmprin activity-blocking antibody (UM-8D6) as described in Materials and methods. The culture media were subjected to immunoblotting using an MMP- $2 \mathrm{mAb}$. The number for anti-emmprin antibody and control IgG indicates the concentrations added to co-cultures $(\mu \mathrm{g} / \mathrm{ml})$. (b) Knockdown of emmprin expression in gastric cancer cell line TMK-1 was analyzed by immunoblotting using E11F4. Short hairpin RNA (shRNA) sequences targeting emmprin mRNA, shBSG, was retrovirally transduced in TMK-1 cells, as shown. Cells were also infected with empty virus (ShLacz) to be used as a control. (c) The cultured media of co-cultures of shBSG or shLacz with ST353 fibroblast were analyzed by immnoblotting using an MMP-2 mAb. Representative examples of three similar results.

(a)

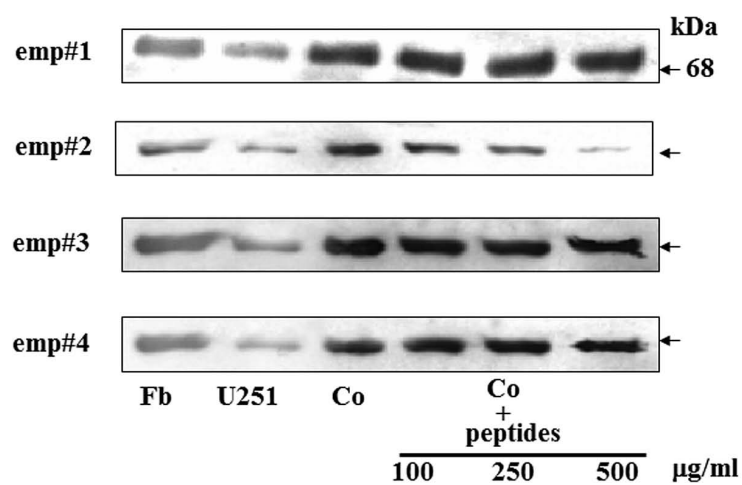

(b)

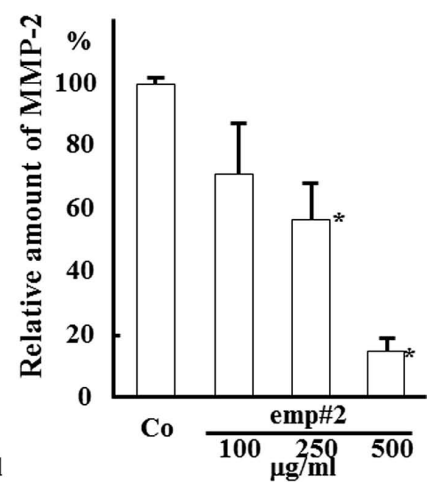

(c)

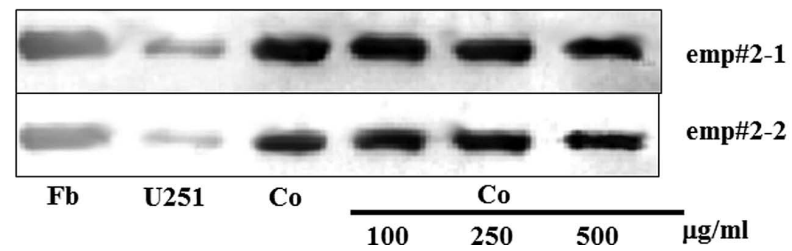

Figure 4. Effect of emmprin peptides on stimulated production of MMP-2 in co-cultures. Co-culture experiments with U251 glioblastoma cells and fibroblast were run in the presence of synthetic peptides emp\#1-4 or emp\#2-1-2 as described in Materials and methods, and their conditioned media were subjected to immunoblotting. (a) Representative immunoblots. (b) Relative protein levels of MMP-2 in co-cultures treated with emp\#2 compared with those in untreated co-cultures. Data are mean \pm SEM $(n=3)$. "P<0.01, compared with the control. (c) Effect of emp\#2-1 and \#2-2 peptides on stimulated production of MMP-2 in co-cultures. Fb, fibroblasts alone; U251, U251 glioblastoma cells alone; Co, co-culture of U251 glioblastoma cells and fibroblasts. Blots in (a) and (c) are representative examples of three similar results.

ments of U251 glioblastoma cells and MBT-3 fibroblasts were run in the presence of four ECI-derived peptides, emp\#1-4. Only the emp\#2 peptide inhibited emmprin-stimulated production of MMP-2 in the co-cultures, while the other peptides showed 
FU-EPS-1

(ES)

A375

(MM)
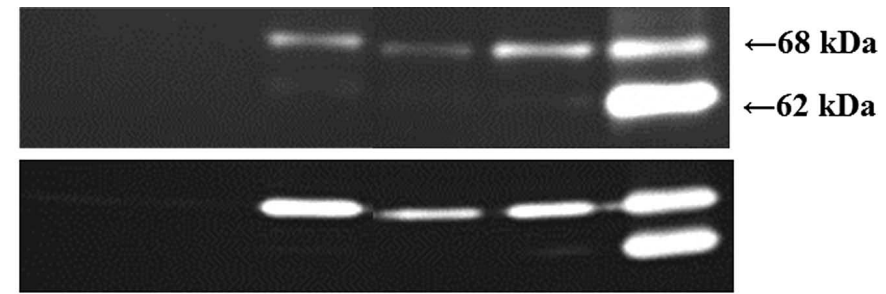

MMP-2 control

$\begin{array}{lcccccc}\text { Fibroblast } & 0.4 & 0 & 0.4 & 0.4 & 0.4 & \\ \text { Tumor cell } & 0 & 0.4 & 0.4 & 0.4 & 0.4 & \left(\times 10^{5}\right) \\ \text { Emp\#2 peptide } & - & - & - & 500 & - & \\ \text { control peptide } & - & - & - & - & 500 & \mu \mathrm{g} / \mathrm{ml}\end{array}$

Figure 5. Reversal of MMP-2 gelatinolytic activity stimulation in co-cultures of FU-EPS-1 epithelioid sarcoma cells or A375 melanoma cells and ST353 fibroblasts by emp\#2 peptide. After co-culture experiments were performed in the presence of the emmprin activity-blocking peptide emp\#2, the culture media were subjected to gelatin zymography. Bands at $68 \mathrm{kDa}$ correspond to the pro-form of MMP-2. The number for fibroblasts and tumor cells indicates the cell number used in co-cultures $\left(\mathrm{x} 10^{5} / \mathrm{ml}\right)$. The number for emp\#2 and control peptides indicates the concentrations added to co-cultures $(\mu \mathrm{g} / \mathrm{ml})$. Representative examples of three similar results.

Table I. Effect of emp \#2 peptides on stimulated production of MMP-2 in co-cultures.

\begin{tabular}{lccc}
\hline Cell type & Tumor origin & Method & $\begin{array}{c}\text { Inhibition of stimulation of } \\
\text { MMP-2 production in co-cultures }\end{array}$ \\
\hline FUEPS-1 & Epithelioid sarcoma & Zymography & $85.5 \pm 4.4 \%$ \\
U251 & Glioblastoma & Immunoblotting & $86.2 \pm 4.6 \%$ \\
A375 & Malignant melanoma & Zymography & $48.3 \pm 11.8 \%$ \\
MT2 & ATL & Immunoblotting & $77.8 \pm 1.1 \%$ \\
RCM-1 & Colon carcinoma & Immunoblotting & $64.7 \pm 12.7 \%^{\mathrm{a}}$ \\
\hline
\end{tabular}

Data are mean $\pm \operatorname{SEM}(\mathrm{n}=3)$. ${ }^{a}$ at $100 \mathrm{mg} / \mathrm{ml}$. ATL, adult T-cell leukemia.

no inhibitory effect (Fig. 4a). The inhibitory activity of emp\#2 peptide was dose-dependent, with approximately 30, 45 and $85 \%$ inhibition at 100, 250 and $500 \mu \mathrm{g} / \mathrm{ml}$, respectively (Fig. 4b).

To determine whether a shorter peptide was still active, the first half (10 aa) and the second half (10 aa) of the emp\#2 peptide were synthesized (emp\#2-1 and emp\#2-2, respectively) for use in the co-culture experiments. However, neither of these two peptides inhibited emmprin-enhanced production of MMP-2 in the co-cultures (Fig. 4c).

We also confirmed the effect of the emp\#2 peptide in co-cultures of other human tumor cells and fibroblasts. Fig. 5 shows representatives gelatin zymography of the conditioned media of co-cultures with emp\#2 peptide. The presence of emp\#2 peptide $(500 \mu \mathrm{g} / \mathrm{ml})$ inhibited the stimulation of MMP-2 production by $85.5 \pm 4.4 \%$ or $77.8 \pm 1.1 \%$ in co-cultures of FU-EPS-1 sarcoma cells or A375 melanoma cells and fibroblasts, respectively, while a control 20 -aa peptide caused no inhibition. Table I summarizes these findings in co-cultures of five human tumor cell lines and fibroblasts. Thus, synthetic ECI-derived emp\#2 peptide, containing a putative $\mathrm{N}$-glycosylation site, significantly inhibited emmprin-stimu- lated production of MMP-2 in co-cultures of various types of tumor cells and fibroblasts.

Effect of emp\#2 on glioblastoma cell invasion through Matrigel enhanced by co-culture with fibroblasts. Next, we investigated the inhibitory effect of emp\#2 peptide on the invasive activity of tumor cells that is promoted by interacting with fibroblasts, probably due to MMP-2 upregulation. In the presence of fibroblasts, the number of invading U251 glioblastoma cells significantly increased (2 times) compared with individual cultures of U251 cells (Fig. 6). Preincubation with emp\#2 peptide of U251 tumor cells suppressed the stimulated invasion of Matrigel in co-cultures by about $65 \%$ (Fig. 6).

\section{Discussion}

The present study demonstrated that synthetic emmprin peptides could inhibit emmprin activity. An ECI-derived peptide, emp\#2, which contains a putative $\mathrm{N}$-glycosylation site sequence, significantly inhibited emmprin-stimulated production of MMP-2 in cocultures of tumor cells and fibro- 


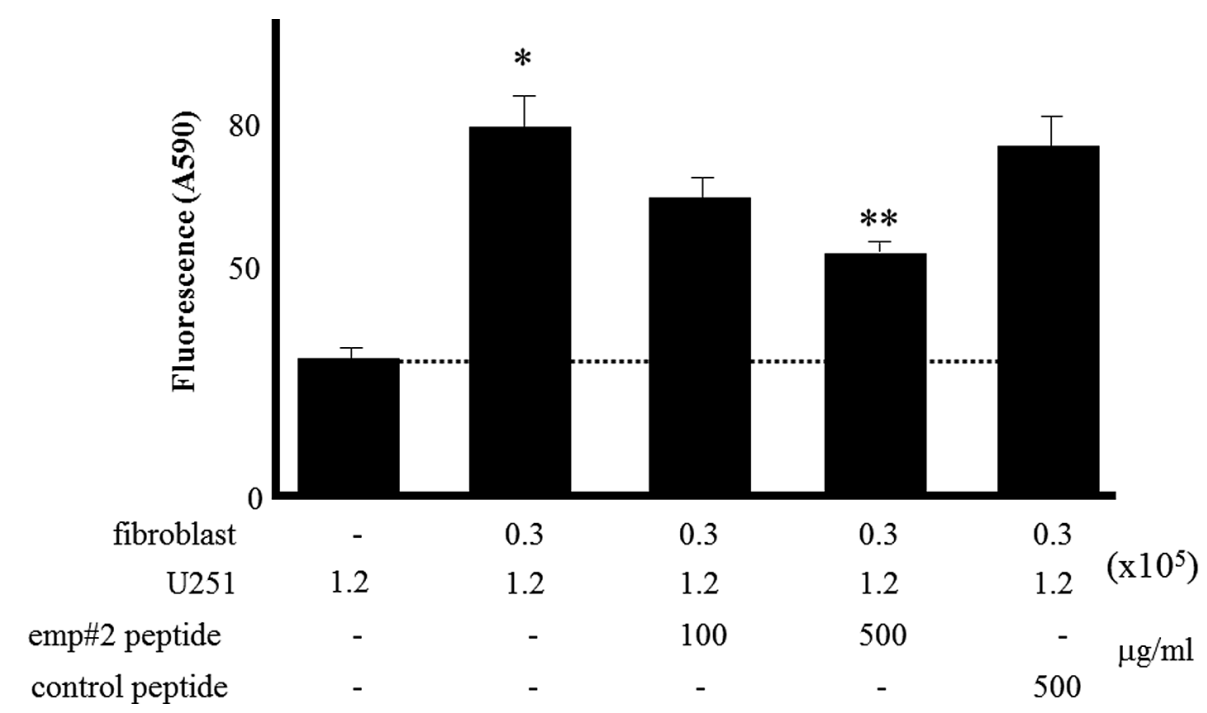

Figure 6. Inhibitory effects of emp\#2 peptide on U251 glioblastoma cell invasive activity in co-culture condition with ST353 fibroblasts. U251 cells were labeled with Dil and preincubated with emp\#2 and control peptides. Invasion assays were then performed for $72 \mathrm{~h}$. The number for fibroblasts and U251 cells indicates the cell number used in co-cultures (x10 $/ \mathrm{ml})$. The number for emp\#2 and control peptides indicates the concentrations added to co-cultures $(\mu \mathrm{g} / \mathrm{ml})$. Data are mean \pm SEM $(n=4)$. ${ }^{*} \mathrm{P}<0.01$, compared with individual cultures (Student's t-test). ${ }^{* *} \mathrm{P}<0.01$, compared with control peptide- treated cells (Student's t-test).

blasts. This peptide was effective on variously glycosylated emmprin molecules expressed on various tumor cells, including carcinoma, sarcoma, melanoma, leukemia and glioma cells. Moreover, the peptide significantly inhibited the invasive activity of glioblastoma cells promoted by interacting with fibroblasts.

We have mainly studied the role of emmprin in heterotypic interactions between tumor cells and fibroblasts, but emmprin is also involved in homophilic interactions between tumor cells (26). Overexpression of emmprin in tumor cells leads to increased MMP production within the same population of cells $(27,28)$. In both types of cellular interactions, emmprin is postulated to work via homophilic interactions between emmprin molecules or through binding to a putative receptor/ acceptor molecule $(20,26)$. In either case, since competitive inhibition by decoy molecules that bind the active site but are themselves functionally inactive could be possible, emp\#2 peptide may act as a decoy. Recently, purified deglycosylated emmprin was shown to antagonize the MMP-1-inducing activity of purified native glycosylated emmprin (26). Moreover, the deglycosylated emmprin extracellular domains including ECI, prepared as an Fc-fusion protein, markedly inhibited not only the homophilic interaction between emmprin molecules, but also the production of MMP-2 by the breast cancer cell line MDA-435 (26). Our study narrowed down the size of decoy molecules to 20 aa long, which is advantageous for their clinical use. Our study is strongly supported by a report by Sato et al (29), in which a synthetic emmprin peptide EM1, composed of the first 18 aa of the emp\#2 peptide, interfered with emmprin-mediated proMMP-1 production in co-cultures of human uterine cervical carcinoma SKG-II cells and human uterine cervical fibroblasts.

Another possible explanation for the emmprin-inhibitory effect of emp\#2 is that emp\#2 may interfere with homophilic or heterophilic cis-interactions between emmprin molecules within the plasma membrane or between emmprin and an unidentified cell surface molecule(s) on tumor cells that is required to bind a putative receptor/acceptor on fibroblasts. This possibility is based on our interesting findings that the inhibitory effect of emp\#2 peptide on emmprin-mediated enhanced MMP-2 production was observed only when the peptide was preincubated with tumor cells, whereas no inhibitory activity was detected when the peptide was preincubated with fibroblasts (data not shown). A previous study associated emmprin with three major types of transporters, CD98 heavy chain (CD98hc), L-type amino acid transporter, ASCT2, and monocarboxylate transporters, in addition to a regulator of cell proliferation (epithelial cell adhesion molecule) (30). Recently, several other molecules have also been reported to associate with emmprin in regulating cancer invasion and migration (31). One or some of these molecules or other as yet undetermined ones may be a target(s) of the emp\#2 peptide. In ongoing studies, we are investigating membrane molecules that complex with emmprin in a manner that is sensitive to inhibition by the emp\#2 peptide.

The third possible inhibitory mechanism could involve the lectin activity of emmprin (32). The fourth Ig-like domain of neural cell adhesion molecule (NCAM) is a lectin domain, and NCAM forms a complex with another neural adhesion molecule L1 via oligomannosidic carbohydrates on L1. The 19-aa peptide that comprises part of the C-type lectin consensus sequence in the fourth Ig-like domain of NACAM interferes with the association between L1 and NCAM (33). ECI of emmprin is homologous to this lectin domain of NCAM, and emmprin was shown to bind the high-mannose-type L3 carbohydrate epitope, leading to emmprin-dependent astrocytic out growth (32). However, it remains to be elucidated whether lectin-type binding via oligomannosidic carbohydrates plays a role in the emmprin-mediated MMP-2 upregulation observed in our tumor cell-fibroblast co-cultures. 
The process of tumor cell invasion and metastasis is very complex, involving not only MMP expression, but also the activation of integrins and other adhesion receptors and the deposition of matrix molecules. The expression of emmprin in association with these factors appears to facilitate the ultimate invasive behavior of several tumors. Emmprin facilitated invasion through a reconstituted basement membrane in human oral squamous cell carcinoma (34) and hepatoma cells (35), while an in vivo study showed that emmprin cDNA-transfected breast cancer cells were considerably more tumorigenic and invasive than plasmid-transfected cancer cells when implanted into mouse (28). Furthermore, downregulation of emmprin via RNA interference reduced tumor cell invasion $(36,37)$, and stimulation of tumor cell invasion by co-culturing tumor cells with fibroblasts was also reported (38). In this latter study, co-cultures of SKG-II cells and fibroblasts exhibited increased in vitro invasive activity of SKG-II cells via increased activation of fibroblast-derived proMMP-2 on the tumor cell surface. Promotion of tumorigenesis by co-injection of emmprin-expressing tumor cells and dermal fibroblasts was also reported (39). In our study, emp\#2 peptide inhibited not only emmprin-mediated MMP-2 upregulation, but also the glioblastoma cell invasion augmented by interacting with fibroblasts.

Emmprin expression is frequently detected in the vast majority of human malignancies (40). Perturbation and inhibition of emmprin-mediated paracrine and autocrine signaling networks by use of competitive peptides such as emp\#2 used in this study could have potential therapeutic value in the prevention of cancer invasion and progression.

\section{Acknowledgements}

The authors thank Ms. M. Onitsuka for the skillful assistance in the cell culture and co-culture experiments.

\section{References}

1. Liotta LA and Stetler-Stevenson WG: Tumor invasion and metastasis: an imbalance of positive and negative regulation. Cancer Res 51: S5054-S5059, 1991.

2. Poulsom R, Pignatelli M, Stetler-Stevenson WG, et al: Stromal expression of $72 \mathrm{kDa}$ type IV collagenase (MMP-2) and TIMP-2 mRNAs in colorectal neoplasia. Am J Pathol 141: 389-396, 1992.

3. Pyke C, Ralfkiaer E, Huhtala P, Hurskainen T, Dano K and Tryggvason K: Localization of messenger RNA for Mr 72,000 and 92,000 type IV collagenases in human skin cancers by in situ hybridization. Cancer Res 52: 1336-1341, 1992.

4. Poulsom R, Hanby AM, Pignatelli M, et al: Expression of gelatinase A and TIMP-2 mRNAs in desmoplastic fibroblasts in both mammary carcinomas and basal cell carcinomas of the skin. J Clin Pathol 46: 429-436, 1993.

5. Sameshima T, Nabeshima K, Toole BP, et al: Glioma cell extracellular matrix metalloproteinase inducer (EMMPRIN) (CD147) stimulates production of membrane-type matrix metalloproteinases and activated gelatinase A in co-cultures with brain-derived fibroblasts. Cancer Lett 157: 177-184, 2000.

6. Koga K, Nabeshima K, Aoki M, et al: Emmprin in epithelioid sarcoma: expression in tumor cell membrane and stimulation of MMP-2 production in tumor-associated fibroblasts. Int J Cancer 120: 761-768, 2007.

7. Nabeshima K, Suzumiya J, Nagano M, et al: Emmprin, a cell surface inducer of matrix metalloproteinases (MMPs), is expressed in T-cell lymphomas. J Pathol 202: 341-351, 2004.

8. Li R, Huang L, Guo H and Toole BP: Basigin (murine EMMPRIN) stimulates matrix metalloproteinase production by fibroblasts. J Cell Physiol 186: 371-379, 2001.
9. Sameshima T, Nabeshima K, Toole BP, et al: Expression of emmprin (CD147), a cell surface inducer of matrix metalloproteinases, in normal human brain and gliomas. Int J Cancer 88: 21-27, 2000.

10. Tang Y, Nakada MT, Kesavan P, et al: Extracellular matrix metalloproteinase inducer stimulates tumor angiogenesis by elevating vascular endothelial cell growth factor and matrix metalloproteinases. Cancer Res 65: 3193-3199, 2005.

11. Baba M, Inoue M, Itoh K and Nishizawa Y: Blocking CD147 induces cell death in cancer cells through impairment of glycolytic energy metabolism. Biochem Biophys Res Commun 374: $111-116,2008$.

12. Su J, Chen X and Kanekura T: A CD147-targeting siRNA inhibits the proliferation, invasiveness, and VEGF production of human malignant melanoma cells by down-regulating glycolysis. Cancer Lett 273: 140-147, 2009.

13. Yang JM, O'Neill P, Jin W, et al: Extracellular matrix metalloproteinase inducer (CD147) confers resistance of breast cancer cells to Anoikis through inhibition of Bim. J Biol Chem 281: 9719-9727, 2006

14. Marieb EA, Zoltan-Jones A, Li R, et al: Emmprin promotes anchorage-independent growth in human mammary carcinoma cells by stimulating hyaluronan production. Cancer Res 64: 1229-1232, 2004

15. Misra S, Ghatak S, Zoltan-Jones A and Toole BP: Regulation of multidrug resistance in cancer cells by hyaluronan. J Biol Chem 278: 25285-25288, 2003.

16. Kuang YH, Chen X, Su J, et al: Proteome analysis of multidrug resistance of human oral squamous carcinoma cells using CD147 silencing. J Proteome Res 7: 4784-4791, 2008.

17. Huet E, Vallee B, Szul D, et al: Extracellular matrix metalloproteinase inducer/CD147 promotes myofibroblast differentiation by inducing alpha-smooth muscle actin expression and collagen gel contraction: implications in tissue remodeling. FASEB J 22: 1144-1154, 2008

18. Biswas C, Zhang Y, DeCastro R, et al: The human tumor cellderived collagenase stimulatory factor (renamed EMMPRIN) is a member of the immunoglobulin superfamily. Cancer Res 55: 434-439, 1995

19. Guo H, Zucker S, Gordon MK, Toole BP and Biswas C: Stimulation of matrix metalloproteinase production by recombinant extracellular matrix metalloproteinase inducer from transfected Chinese hamster ovary cells. J Biol Chem 272: 24-27, 1997.

20. Toole BP: Emmprin (CD147), a cell surface regulator of matrix metalloproteinase production and function. Curr Top Dev Biol 54: 371-389, 2003.

21. Miyoshi I, Kubonishi I, Yoshimoto S and Shiraishi Y: A T-cell line derived from normal human cord leukocytes by co-culturing with human leukemic T-cells. Gann 72: 978-981, 1981.

22. Nishio J, Iwasaki H, Nabeshima K, et al: Establishment of a new human epithelioid sarcoma cell line, FU-EPS-1: molecular cytogenetic characterization by use of spectral karyotyping and comparative genomic hybridization. Int J Oncol 27: 361-369, 2005.

23. Ellis SM, Nabeshima $K$ and Biswas C: Monoclonal antibody preparation and purification of a tumor cell collagenase-stimulatory factor. Cancer Res 49: 3385-3391, 1989.

24. Niiya D, Egawa N, Sakamoto T, et al: Identification and characterization of Lutheran blood group glycoprotein as a new substrate of membrane-type 1 matrix metalloproteinase 1 (MT1-MMP): a systemic whole cell analysis of MT1-MMP-associating proteins in A431 cells. J Biol Chem 284: 27360-27369, 2009.

25. Shulby SA, Dolloff NG, Stearns ME, Meucci O and Fatatis A: CX3CR1-fractalkine expression regulates cellular mechanisms involved in adhesion, migration, and survival of human prostate cancer cells. Cancer Res 64: 4693-4698, 2004

26. Sun J and Hemler ME: Regulation of MMP-1 and MMP-2 production through CD147/extracellular matrix metalloproteinase inducer interactions. Cancer Res 61: 2276-2281, 2001.

27. Caudroy S, Polette M, Nawrocki-Raby B, et al: EMMPRINmediated MMP regulation in tumor and endothelial cells. Clin Exp Metastasis 19: 697-702, 2002.

28. Zucker S, Hymowitz M, Rollo EE, et al: Tumorigenic potential of extracellular matrix metalloproteinase inducer. Am J Pathol 158: 1921-1928, 2001.

29. Sato T, Ota T, Watanabe M, Imada K, Nomizu M and Ito A: Identification of an active site of EMMPRIN for the augmentation of matrix metalloproteinase-1 and -3 expression in a co-culture of human uterine cervical carcinoma cells and fibroblasts. Gynecol Oncol 114: 337-342, 2009. 
30. Xu D and Hemler ME: Metabolic activation-related CD147CD98 complex. Mol Cell Proteomics 4: 1061-1071, 2005.

31. Zhao P, Zhang W, Tang J, et al: Annexin II promotes invasion and migration of human hepatocellular carcinoma cells in vitro via its interaction with HAb18G/CD147. Cancer Sci 101: 387-395, 2010.

32. Heller M, von der Ohe M, Kleene R, Mohajeri MH and Schachner M: The immunoglobulin-superfamily molecule basigin is a binding protein for oligomannosidic carbohydrates: an anti-idiotypic approach. J Neurochem 84: 557-565, 2003.

33. Horstkorte R, Schachner M, Magyar JP, Vorherr T and Schmitz B: The fourth immunoglobulin-like domain of NCAM contains a carbohydrate recognition domain for oligomannosidic glycans implicated in association with L1 and neurite outgrowth. J Cell Biol 121: 1409-1421, 1993.

34. Bordador LC, Li X, Toole B, et al: Expression of emmprin by oral squamous cell carcinoma. Int J Cancer 85: 347-352, 2000.

35. Jiang JL, Zhou Q, Yu MK, Ho LS, Chen ZN and Chan HC: The involvement of $\mathrm{HAb} 18 \mathrm{G} / \mathrm{CD} 147$ in regulation of store-operated calcium entry and metastasis of human hepatoma cells. J Biol Chem 276: 46870-46877, 2001.
36. Zou W, Yang H, Hou X, Zhang W, Chen B and Xin X: Inhibition of CD147 gene expression via RNA interference reduces tumor cell invasion, tumorigenicity and increases chemosensitivity to paclitaxel in HO-8910pm cells. Cancer Lett 248: 211-218, 2007.

37. Chen X, Lin J, Kanekura T, et al: A small interfering CD147targeting RNA inhibited the proliferation, invasiveness, and metastatic activity of malignant melanoma. Cancer Res 66: 11323-11330, 2006.

38. Sato T, Sakai T, Noguchi Y, Takita M, Hirakawa S and Ito A: Tumor-stromal cell contact promotes invasion of human uterine cervical carcinoma cells by augmenting the expression and activation of stromal matrix metalloproteinases. Gynecol Oncol 92: 47-56, 2004.

39. Rosenthal EL, Vidrine DM and Zhang W: Extracellular matrix metalloprotease inducer stimulates fibroblast-mediated tumor growth in vivo. Laryngoscope 116: 1086-1092, 2006.

40. Riethdorf S, Reimers N, Assmann V, et al: High incidence of EMMPRIN expression in human tumors. Int J Cancer 119: 1800-1810, 2006 